\title{
Disponibilidade de fósforo em sítios de terra preta arqueológica em Oriximiná, Brasil
}

Grande parte dos solos amazônicos geralmente são ácidos, com capacidade de troca de cátions e fertilidade baixas, o que os torna limitante para o aumento da produtividade e sustentabilidade dos sistemas agrícolas nesta região. Devido a isso, agricultores familiares estão utilizando áreas de Terra Preta Arqueológica (TPA) para os seus cultivos, pois estas apresentam elevados teores de nutrientes, principalmente fósforo e cálcio (P e Ca). As TPA são decorrentes de atividades humanas pretéritas, portanto, são antigos assentamentos indígenas caracterizados como sítios arqueológicos. Neste sentido, o objetivo no trabalho foi analisar a disponibilidade de $\mathrm{P}$ nos solos de cinco sítios arqueológicos no Município de Oriximiná, Brasil. Foram avaliadas amostras de TPA nas profundidades 0-0,20 e 0,20$0,40 \mathrm{~m}$ dos sítios arqueológicos (Maracajá, Faissal, Praia, Juquirizinhos I e II). Os teores de P disponível foram elevados, variando de 4 a 304 mg kg-1. O sítio arqueológico que apresentou o maior teor médio foi o Juquirizinho l, variando de 17 a $202 \mathrm{mg} \mathrm{kg-1} \mathrm{de} \mathrm{P} \mathrm{e} \mathrm{o} \mathrm{que} \mathrm{apresentou} \mathrm{menor} \mathrm{teor} \mathrm{médio} \mathrm{foi} \mathrm{o} \mathrm{Praia,} \mathrm{variando}$ de 5 a $20 \mathrm{mg} \mathrm{kg-1}$ de P. Houve grande variação nos teores de P entre os sítios, com destaque para o Juquirizinho I, que apresentou valores entre 17 a $202 \mathrm{mg} \mathrm{kg}-$ 1. Quatro dos cinco sítios avaliados apresentaram alta disponibilidade de $P$, mas algumas áreas amostradas apresentaram valores abaixo de 30 mg kg-1, neste sentido não apresentaram horizonte A antrópico. Houve grande variação dos teores de fósforo em profundidade, considerando o mesmo sítio arqueológico.

Palavras-chave: Amazônia; Horizonte Au; Populações pré-colombianas; Terra preta de índio.

\section{Phosphorus availability in sites archeological black earth in Oriximiná, Brazil}

\begin{abstract}
Amazonian soils are generally acidic, with low cation exchange capacity and low fertility, which makes them limiting for increasing productivity and sustainability of agricultural systems in this region. Because of this, many family farmers are using archeological black earth (ABE) areas for their crops, as these have high levels of nutrients, mainly phosphorus (P). In this sense, the objective in the work was to analyze the availability of $P$ in the soils of five archaeological sites in the Municipality of Oriximiná, Brazil. ABE samples were evaluated at depths $0-0.20$ and $0.20-0.40 \mathrm{~m}$ from the archaeological sites (Maracajá, Faissal, Praia, Juquirizinhos I and II). The levels of available P were high, ranging from 4 to $304 \mathrm{mg} \mathrm{kg-1.} \mathrm{The} \mathrm{archeological} \mathrm{site} \mathrm{that} \mathrm{had} \mathrm{the} \mathrm{highest} \mathrm{average} \mathrm{content} \mathrm{was} \mathrm{Juquirizinho} \mathrm{I,} \mathrm{rangin}$ from 17 to $202 \mathrm{mg} \mathrm{kg-} 1 \mathrm{P}$ and the one with the lowest average content was Praia, ranging from 5 to $20 \mathrm{mg} \mathrm{kg}-1$ of $\mathrm{P}$. There was great variation in the levels of $\mathrm{P}$ between the sites, with emphasis on Juquirizinho I, which presented values between 17 to $202 \mathrm{mg} \mathrm{kg}-1$. Four of the five sites evaluated showed high availability of $\mathrm{P}$, but some areas sampled showed values below $30 \mathrm{mg} \mathrm{kg}-1$, in this sense, they did not present an anthropic A horizon. There was a great variation in the levels of phosphorus in depth, considering the same archaeological site.
\end{abstract}

Keywords: Amazon; Horizont Au; Pre-Colombian populations; Black Indian earth

Topic: Ciências do Solo

Reviewed anonymously in the process of blind peer
Received: 02/01/2021

Approved: 25/01/2021
Lucas Santos da Silva (iD)

Universidade Federal do Oeste do Pará, Brasil http://lattes.cnpq.br/6800229989379325 http://orcid.org/0000-0001-5612-9311 lucassilva.iap@gmail.com

Iolanda Maria Soares Reis (iD) Universidade Federal do Oeste do Pará, Brasil http://lattes.cnpq.br/9591454475619777 http://orcid.org/0000-0001-6619-0730 iolandareis@outlook.com

Mateus Alves de Sousa (iD

Universidade Federal do Oeste do Pará, Brasil http://lattes.cnpq.br/7484606325846483 http://orcid.org/0000-0002-9696-743X mateussica@gmail.com

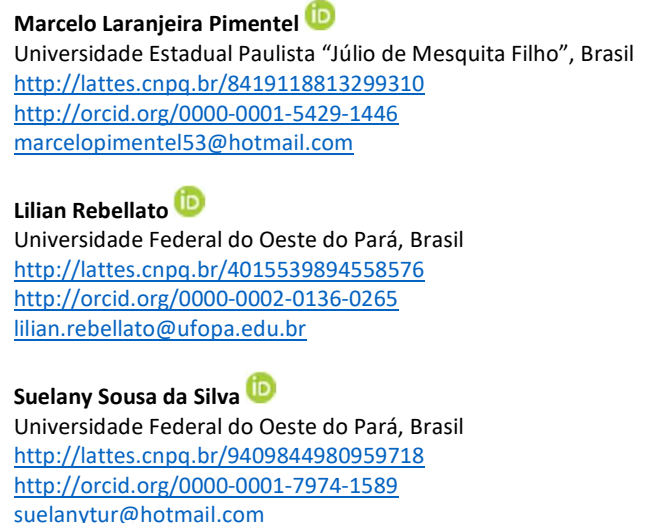

Ulisses Sidnei da Conceição Silva (iD Universidade Federal do Oeste do Pará, Brasil http://lattes.cnpq.br/2371065316621348 http://orcid.org/0000-0002-7650-8375 usc.silva@gmail.com
Referencing this:

SILVA, L. S.; REIS, I. M. S.; SOUSA, M. A.; PIMENTEL, M. L.; REBELLATO, L.; SILVA, S. S.; SILVA, U. S. C.. Disponibilidade de fósforo em sítios de terra preta arqueológica em Oriximiná, Brasil. Revista Ibero Americana de Ciências Ambientais, v.12, n.1, p.23-30, 2021. DOI: http://doi.org/10.6008/CBPC2179-6858.2021.001.0003 


\section{INTRODUÇÃO}

A maioria dos solos agricultáveis amazônicos são ácidos, com baixa capacidade de trocas de cátions e baixa fertilidade, sendo um fator limitante para o aumento da produtividade e sustentabilidade de sistemas de produção agrícola na região (CUNHA, 2005; GLASER et al., 2012; SCHELLEKENS et al., 2017). Devido a isso, agricultores familiares (SOARES et al., 2018) e comunidades tradicionais (SOUSA et al., 2020), estão utilizando áreas de Terra Preta de Arqueológica (TPA) para alguns de seus cultivos, por apresentarem elevados teores de nutrientes, principalmente $\mathrm{Ca}^{2+}$ e $\mathrm{P}$.

As TPAs são decorrentes de atividades humanas pretéritas, cuja a queima incompleta de matéria orgânica preservou muitos desses dejetos (GLASER, 2003). Sua ocorrência é comum em toda Amazônia, cujas atividades cotidianas alteraram os solos, dando origem à mundialmente conhecida Terra Preta de Índio (TPI) ou Terra Preta Arqueológica (TPA) (GLASER et al., 2012; SANTOS et al., 2013).

Normalmente, as TPA's ocupam pequenas áreas, de dois a cinco hectares (GLASER et al., 2012; PESSOA JÚNIOR et. al., 2012), possuindo grande importância histórica e enorme valor nutricional. São encontradas em diversos tipos de solos, tais como Latossolos, Argissolos, Cambissolos e Nitossolos (CUNHA, 2005). As TPA's ocorrem tanto no Brasil quanto na Bolívia, Peru, Suriname, Guiana, Colômbia (SOMBROEK, 2003).

Segundo o Sistema Brasileiro de Classificação de Solos 5ạ edição, TPA's são solos com horizonte A antrópico (Au), ou seja, formados ou modificados pelo uso contínuo de atividades humanas, estando ou não misturado com material orgânico, com presença de artefatos cerâmicos e/ou líticos, ossos, conchas ou vestígios de ação do fogo (carvão e cinzas), espessura maior ou igual a 0,2 m e possui conteúdo de fósforo (P) extraível (com solução Mehlich-1) maior do que $30 \mathrm{mg} \mathrm{kg}^{-1}$ de solo (SANTOS et al., 2018). Sendo fundamental conhecer estes solos para identificação e conservação dos mesmos.

Neste sentido, o objetivo no trabalho foi analisar a disponibilidade de $\mathrm{P}$ nos solos de cinco sítios arqueológicos no Município de Oriximiná, Brasil.

\section{MATERIAIS E MÉTODOS}

\section{Área de estudo}

O trabalho foi realizado com parceria do Projeto 'Geoarqueologia na Amazônia', cadastrado na Universidade Federal do Oeste do Pará e financiado pelo CNPQ. A área de estudo foi a Floresta Nacional Saracá-Taquera, margem direita e, na Reserva Biológica Rio Trombetas, na margem esquerda, município de Oriximiná, Pará, Brasil. Habitada por comunidades remanescentes de quilombos, o que favoreceu as práticas tradicionais de manejo da paisagem, bem como a preservação de recursos naturais e da identidade cultural e sua história de ocupação. Essa região apresenta, em sua grande maioria, solos das classes Argissolo, Latossolo e Espodossolo, mas os sítios arqueológicos estão localizados em uma área classificada como associação de solos Latossolos Amarelos distrófico + Argissolo Vermelho-Amarelo distrófico + Plintossolo Háplico distrófico, em relevo abaciado, próximos aos cursos d'agua (Figura 1). 
A vegetação predominante é a Floresta Ombrófila Densa. De acordo com a estação meteorológica de Óbidos, Pará, essa região apresenta um clima úmido, com precipitação anual média de 2.379 mm e temperatura média anual de $27,2{ }^{\circ} \mathrm{C}$ (INMET, 2019), o que contribui substancialmente para a intensificação do intemperismo e consequente acidificação dos solos, tornando os solos de TPA um diferencial de fertilidade na região. Os picos de precipitação ocorrem entre os meses de março e maio, o que corresponde ao período chuvoso amazônico, conforme demonstrado por dados, considerando um período de 2010 a 2019, da estação meteorológica de Óbidos, a mais próxima da região (Figura 2). A área de estudo pertence à mesorregião Baixo Amazonas e a microrregião de Óbidos, no extremo Noroeste do Estado do Pará, Brasil (ARAÚJO, 2013).
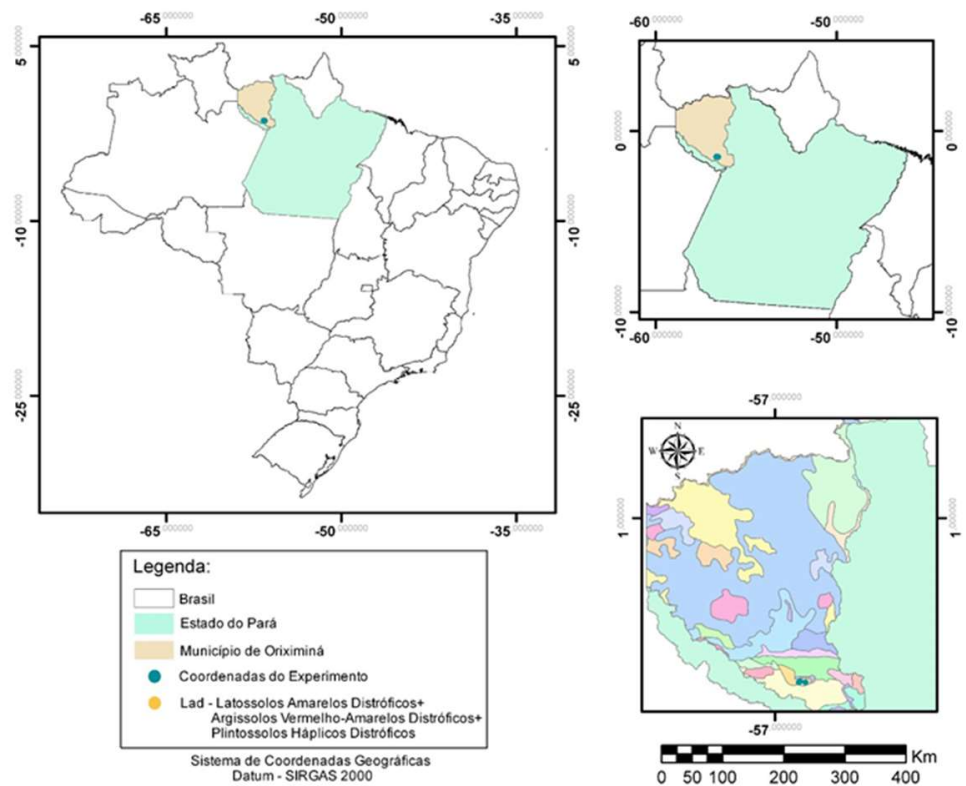

Figura 1: Mapa de localização dos sítios arqueológicos e mapa de solos, em área de terra preta arqueológica, Oriximiná, Pará, Brasil. Software ArcGis versão 10.8, base de dados/Instituto Brasileiro de Geografia e Estatística (2019).

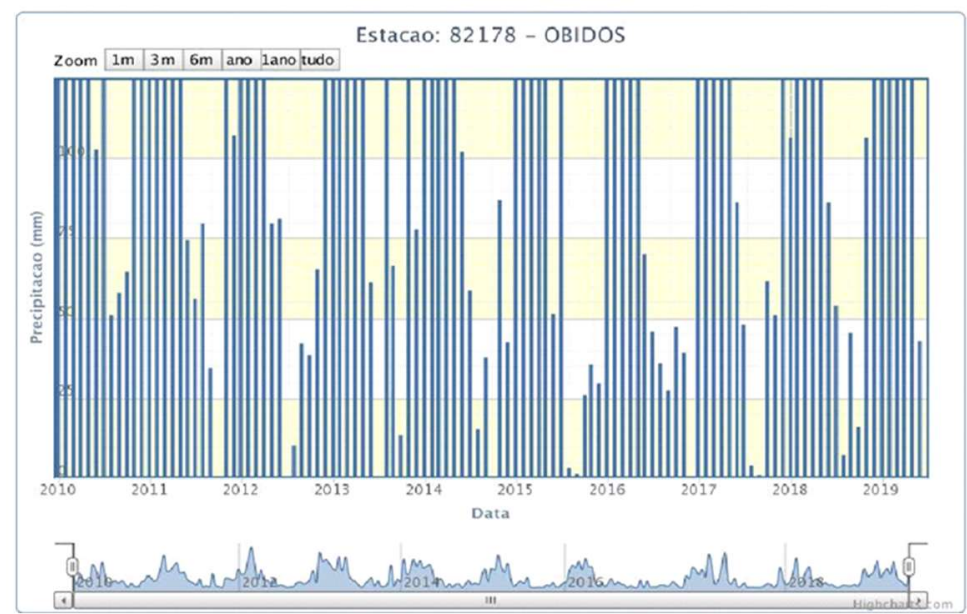

Firgura 2: Precipitação pluviométrica da microrregião de Óbidos, no período de janeiro de 2010 a junho de 2019. Fonte: INMET (2019).

\section{Coleta de solo}

A prospecção ocorreu em cinco sítios arqueológicos: Maracajá, Praia, Faissal, Juquirizinho I e Juquirizinho II de TPA, situados na Floresta Nacional Saracá-Taquera, margens direita e esquerda do rio 
Trombetas, município de Oriximiná. As amostras foram coletadas nas profundidades 0-0,20, 0,20-0,40 m, com auxílio de trado holandês, totalizando 54 amostras de solo (seis do Maracajá, seis do Faissal, seis do Juquirizinho I, vinte do Praia e dezesseis do Juquirizinho II), que posteriormente foram encaminhadas ao Laboratório de Qualidade do Solo da Universidade Federal do Oeste do Pará.

\section{Análise da disponibilidade de Fósforo}

As amostras de solo foram secas ao ar, destorroadas e peneiradas à $2 \mathrm{~mm}$ de abertura de malha para obtenção de Terra Fina Seca ao Ar (TFSA). Para análise de fósforo disponível foi utilizado metodologias compiladas em Teixeira et al. (2017). Para extração dos teores de P foi usada a solução extratora dupla ácido ( $\mathrm{HCl} 0,05 \mathrm{~mol} \mathrm{~L}^{-1}$ e $\mathrm{H}_{2} \mathrm{SO}_{4}$ 0,0125 $\mathrm{mol} \mathrm{L}^{-1}$ ) e para determinação, o método de azul de molibidato, utilizando solução ácida de molibidato de amônio e ácido ascórbico, como agente redutor, a leitura das amostras foram com auxílio de espectrofotômetro/UV-Vis com comprimento de onda de $660 \mathrm{~nm}$.

\section{Análise estatístico}

A partir dos dados de P disponível, foram realizados a estatística descritiva e análise com carta de controle de dados. A carta controle é um instrumento de análise que possibilita monitorar o desempenho de um processo de medição. Os gráficos produzidos determinam estatisticamente uma faixa denominada limites de controle, que é limitada por uma linha superior (limite superior de controle-LSC) e uma linha inferior (limite inferior de controle-LIC), além de uma linha central (limite central-LC). Quando todos os pontos amostrais estiverem dispostos dentro dos limites de controle de forma aleatória, é considerado que o processo está "sob controle". No entanto, se um (ou mais) ponto(s) estiver(em) disposto(s) fora dos limites de controle, há evidência de que o processo está "fora de controle", e que investigação e ação(ões) corretiva(s) são necessárias para detectar e eliminar a(s) causa(s) especiais no processo (OLIVEIRA et al., 2013). Todas as análises foram com auxílio do software Minitab 19, versão-pró.

\section{RESULTADOS E DISCUSSÃO}

Os teores de $\mathrm{P}$ disponível foram elevados, porém muito dispersos, variando no sítio Maracajá de 4 a $304 \mathrm{mg} \mathrm{kg}^{-1}$, Faissal de 20 a $154 \mathrm{mg} \mathrm{kg}^{-1}$, Praia de 5 a $20 \mathrm{mg} \mathrm{kg}^{-1}$, Juquirizinho I variou de 17 a $202 \mathrm{mg} \mathrm{kg}^{-1}$ e Juquirizinho II de 4 a $174 \mathrm{mg} \mathrm{kg}^{-1}$.

Os sítios que apresentaram maiores teores de P disponíveis foram o Juquirizinho I (média de 108 mg $\mathrm{kg}^{-1}$ na profundidade 0,0-0,20 m e $124 \mathrm{mg} \mathrm{kg}^{-1}$ na profundidade 0,20-0,40 m) e Faissal (média de $87 \mathrm{mg} \mathrm{kg}$ ${ }^{-1}$ na profundidade $0,0-0,20 \mathrm{~m} \mathrm{e} 99 \mathrm{mg} \mathrm{kg}^{-1}$ na profundidade $0,20-0,40 \mathrm{~m}$ ).

Os sítios que apresentaram menores valores foram Praia (média de $10 \mathrm{mg} \mathrm{kg}^{-1}$ na profundidade 0,0 0,20 m e $16 \mathrm{mg} \mathrm{kg}^{-1}$ na profundidade 0,20-0,40 m) e Maracajá (média de $32 \mathrm{mg} \mathrm{kg}^{-1}$ na profundidade 0,0$0,20 \mathrm{~cm}$ e $112 \mathrm{mg} \mathrm{kg}^{-1}$ na profundidade 0,20-0,40 m). Esses valores podem ser considerados altos comparados aos de Soares (2007), onde analisou solos de TPI no município de Rio Preto da Eva, Amazonas, sendo encontrado teores de $\mathrm{P}$ disponível, variando de 1 a $18 \mathrm{mg} \mathrm{kg}^{-1}$. 
Resultados semelhantes foram encontrados por Viana et al. (2016), em sítios arqueológicos no município de Novo Aripuanã, Amazonas, onde os teores de P disponíveis variaram de 8 a $135 \mathrm{mg} \mathrm{kg}^{-1}$. Assim como por Campos et al. (2011), analisando sítios arqueológicos no município de Manicoré, Amazonas, região do Rio Madeira, os quais encontraram teores de $\mathrm{P}$ variando de 15 a $230 \mathrm{mg} \mathrm{kg}^{-1}$.

Em contrapartida, os valores encontrados no sítio Praia e Maracajá, podem ser considerados baixos quando se compara com os sítios avaliados por Silva et al. (2011) e Barros et al. (2012), os quais encontraram teores de P disponível variando de 23 a $955 \mathrm{mg} \mathrm{kg}^{-1}$ e 34 a $242 \mathrm{mg} \mathrm{kg}^{-1}$, respectivamente. Santos et al. (2013), avaliando sete perfis de TPA's na região sul do Estado do Amazonas, encontraram nas profundidades entre 0 e 0,50 m, teores de $P$ variando de $48 \mathrm{mg} \mathrm{kg}^{-1}$ a $517 \mathrm{mg} \mathrm{kg}^{-1}$. Valores maiores foram encontrados por Silva (2006) e Falcão et al. (2006), nos quais os teores variaram de 214 a $307 \mathrm{mg} \mathrm{kg}^{-1}$ e 426 a $660 \mathrm{mg} \mathrm{kg}$ -1 , respectivamente.

De acordo com Cunha et al. (2009), os solos de TPI nos municípios de Humaitá, Apuí, Novo Aripuanã, Manicoré, Rio Preto da Eva, Iranduba, Novo Airão e Açutuba, Amazonas, apresentam fertilidade natural superior, estes estudos encontraram teores de P variando de $116 \mathrm{mg} \mathrm{kg}^{-1} \mathrm{em}$ áreas de TPI sob floresta, 290,5 $\mathrm{mg} \mathrm{kg}{ }^{-1}$ em TPI cultivada e 4,7 mg kg-1 em áreas não antropogênicas.

A estatística descritiva (Tabela 1) apresentou média dos teores de fósforo na profundidade $0,0-0,20$ $\mathrm{m}$ de $41,81 \mathrm{mg} \mathrm{kg}^{-1}$ e na 0,20-0,40 m de $64,4 \mathrm{mg} \mathrm{kg}^{-1}$, havendo assim diferenças entre as profundidades.

Tabela 1: Estatística descritiva dos teores de fósforo $(P)$, nas profundidades $0,0-0,20$ e $0,20-0,40$ m, município de Oriximiná, Pará, Brasil.

\begin{tabular}{llllll}
\hline Profundidades & Número de amostras & Média & Desvio Padrão & Mínimo & Máximo \\
\hline P $\left.0,0-0,20{\mathrm{~m}\left(\mathrm{~m} \mathrm{~kg}^{-1}\right)}^{-1}\right)$ & 27 & 41,81 & 42,09 & 5,86 & 147,44 \\
\hline P $0,20-0,40{\mathrm{~m}\left(\mathrm{mg} \mathrm{kg}^{-1}\right)}^{27}$ & 64,40 & 80,30 & 1,80 & 304,60
\end{tabular}

A maioria dos sítios arqueológicos, na profundidade $0,20-0,40 \mathrm{~m}$, apresentam maiores teores de $\mathrm{P}$ disponível. Isso pode ser explicado pela deposição não uniforme dos dejetos domésticos das populações précolombianas, assim como a preservação do $\mathrm{P}$ em camadas mais profundas. Assim como fósforo outras características da TPA são preservadas ao longo dos anos. De acordo com Sousa et al. (2020), a MOS também é preservada em solos de TPA's mesmo quando manejado de forma diferenciada.

A Carta de Controle X-barra da profundidade 0,0-0,2 m (Figura 3), apresentou Limite Superior de Controle (LSC) de 129,40 $\mathrm{mg} \mathrm{kg}^{-1}$, Limite Inferior de Controle (LIC) de $-45,8 \mathrm{mg} \mathrm{kg}^{-1}$ e média de controle (X) de $41,80 \mathrm{mg} \mathrm{kg}^{-1}$. Importante ressaltar que dados de disponibilidade não se apresentam em valores negativos. Os dados analisados apresentam certa estabilidade de controle e apenas uma amostra está acima do limite superior e 11 amostras sequencialmente estão abaixo da média de controle, havendo assim, causa de variação baixa e especial, como a análise é apenas expositiva não foi necessário eliminar a amostra.

O sítio Faissal apresentou no ponto 1 (FP 1) o maior teor de P entre todas as amostras da profundidade em estudo, estando assim, acima do limite superior. Outra causa da variação especial é o fato de todas as amostras do sítio Praia ( $\mathrm{P}$ ) possuírem teores de $\mathrm{P}$ menores que a média de controle, que é de $41,80 \mathrm{mg} \mathrm{kg}^{-1}$, o que pode ser explicado pelo fato dos solos desse sítio encontrarem-se em ambiente de praia, apresentando textura arenosa, sofrendo assim, translocação e/ou lixiviação do nutriente (MAPA, 
2016). Nota-se também que dezesseis amostras apresentaram valores inferiores ao mínimo exigido pelo Sistema Brasileiro de Classificação de Solos (SiBCS), sendo de $30 \mathrm{mg} \mathrm{kg}^{-1}$, descumprindo um dos critérios para ser classificado como horizonte Au (SANTOS et al., 2018).

A Carta de Controle X-barra na profundidade 0,2 - 0,4 m (Figura 4), possui LSC 236,1 mg kg ${ }^{-1}$, LIC 107,3 $\mathrm{mg} \mathrm{kg}^{-1}$ e $X 64,40 \mathrm{mg} \mathrm{kg}^{-1}$. Neste gráfico é possível observar a estabilidade de controle, até certo ponto, devido a uma amostra está acima do LSC e dez amostras sequencialmente estarem abaixo da $X$, existindo causa de variação especial. A existência dessa causa de variação especial é devida ao ponto 2 do sítio Maracajá (MP 2) apresentar teor de P muito elevado quando comparado aos demais pontos, ficando acima do LSC.

Outra observação importante ocorre no sítio Praia, este apresenta teores de P extremamente baixos em relação à $X$, o que pode ser explicado por estar localizado em áreas mais próximas ao rio, onde o relevo predominante é o de planície, sendo mais abaciado, contribuindo para uma maior infiltração da água e, consequentemente maior translocação dos nutrientes.

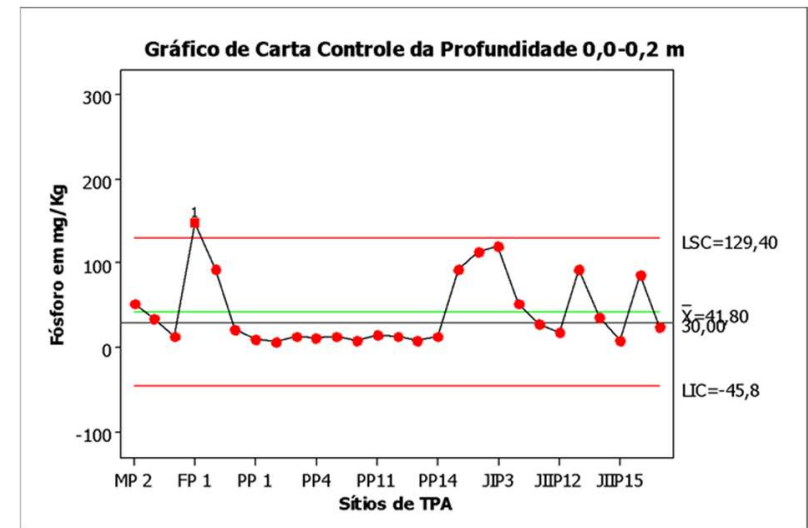

Figura 3: Carta de Controle $X$ - Barra dos teores de Fósforo na profundidade 0,0-0,2 m. LSC (Limite Superior de Controle), $X$ (média de controle), LIC (Limite Inferior de Controle) e $30 \mathrm{mg} \mathrm{kg}^{-1}$ (menor teor de Fósforo exigido pelo SiBCS para TPA).

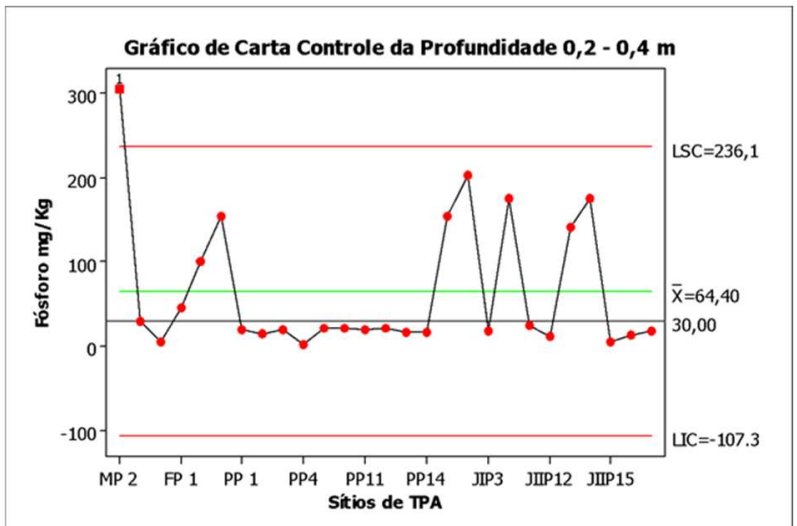

Figura 4: Carta de Controle X-barra dos teores de Fósforo na profundidade 0,2-0,4 m. LSC (Limite Superior de Controle), LIC (Limite Inferior de Controle), $X$ (Média de Controle) e $30 \mathrm{mg} \mathrm{kg}^{-1}$ (menor teor de Fósforo exigido pelo SiBCS para TPA).

Nesta profundidade, 15 amostras apresentaram teores de P abaixo do limite mínimo estabelecido pelo SiBCS, para ser considerado como horizonte Au, descumprindo assim um dos critérios de classificação (SANTOS et al., 2018).

\section{CONCLUSÕES}

Quatro dos cinco sítios avaliados apresentaram alta disponibilidade de fósforo, sendo que teores de elevados de fósforo são determinantes para classificar horizontes A antrópicos. Contudo, algumas áreas amostradas apresentaram valores abaixo de $30 \mathrm{mg} \mathrm{kg}^{-1}$, neste sentido não apresentam horizonte $A$ antropogênico. Houve grande variação dos teores de fósforo em profundidade, considerando o mesmo sítio arqueológico. 


\section{REFERÊNCIAS}

ARAÚJO, C. F.. Estoque de carbono e nitrogênio em terra preta e terra mulata na Reserva Biológica do Rio Trombetas, Oriximiná/Pa. Dissertação (Mestrado em Agricultura no Trópico Úmido) - Instituto Nacional de Pesquisas da Amazônia, Manaus, 2013.

BARROS, K. R. M.; LIMA, H. V.; CANELLAS, L. P.; KERN, D. C.. Fracionamento químico da matéria orgânica e caracterização física de Terra Preta de Índio. Revista de Ciências Agrárias, Belém, v.55, n.1, p.44-51, 2012. DOI:

http://dx.doi.org/10.4322/rca.2012.037

CAMPOS, M. C. C.; RIBEIRO, M. R.; JÚNIOR, V. S. S.; FILHO, M. R. R.; SOUZA, R. V. C. C.; ALMEIDA, M. C.. Caracterização e classificação de terras pretas arqueológicas na Região do Médio Rio Madeira. Bragantia, Campinas, v.70, n.3, p.598609, 2011. DOI: https://doi.org/10.1590/S0006$\underline{87052011000300016}$

CUNHA, T. J. F.. Ácidos húmicos de solos escuros da Amazônia (Terra preta de índio). Tese (Doutorado em Agronomia, Ciência do Solo) - Universidade Federal Rural do Rio de Janeiro, Seropédica, 2005.

CUNHA, T. J. F.; MADARI, B. E.; CANELLAS, L. P.; RIBEIRO, L. P.; BENITES, V. M.; SANTOS, G. A.. Matéria Orgânica e fertilidade de solos antropogênicos (Terra Preta de Índio) da Bacia Amazônica brasileira. Revista Brasileira de Ciência do Solo, Viçosa, v.33, p.85-93. 2009. DOI: https://doi.org/10.1590/S0100-06832009000100009

FALCÃO, N.; MOREIRA, A.; COMENFORD, N. B.. A Fertilidade dos Solos de Terra Preta de Índio da Amazônia Central. In: TEIXEIRA, W. G.. As terras Pretas de Índio da Amazônia: Sua Caracterização e Uso deste Conhecimento na Criação de Novas Áreas. Rio de Janeiro: EMBRAPA, 2009.

GLASER, B.; GUGGENBERGER, G.; ZECH, W.; RUIVO, M. L.. Soil organic matter stability in Amazonian Dark earths. In: LEHMANN, J.; KERN, D. C.; GLASER, B.; WOODS, W. I.. Amazonia Dark earths: Origin, properties and management. Dordrecht, Kluwer Academic Publishers, 2003. p.141-158.

GLASER, B.; BIRK, J. J.. State of the scientific knowledge on properties and genesis of Anthropogenic Dark Earths in Central Amazonia (Terra Preta de Índio). Geochimica et Cosmochimica Acta, v.82, p.39-51, 2012. DOI: https://doi.org/10.1016/i.gca.2010.11.029

INMET. Instituto Nacional de Meteorologia. Estações meteorológicas. INMET, 2019.

IBGE. Instituto Brasileiro de Geografia e Estatística. Base de dados. IBGE, 2019.

MAPA. Ministério da Agricultura, Pecuária e Abastecimento. Fósforo. Brasília: MAPA, 2016.

OLIVEIRA, C. C.; GRANATO, D.; CARUSO, M. S. F.; SAKUMA, A. M.. Manual para elaboração de cartas de controle para monitoramento de processos de medição quantitativos em laboratórios de ensaio. São Paulo: Instituto Adolfo Lutz, 2013.
PESSOA JUNIOR, E. S. F.; SOUZA, W. B.; SOUZA, K. S.; PIO, M. C. S.; SANTANA, G. P.. Terra Preta de Índio na região amazônica. Scientia Amazônia, Manaus, v.1, n.1, 2012.

SANTOS, H. G.; JACOMINE, P. K. T.; ANJOS, L. H. C.; OLIVEIRA, V. A.; LUMBRERAS, J. F.; COELHO, M. R.; ALMEIDA, J. A.; ARAÚJO FILHO, J. C.; OLIVEIRA, J. B.; CUNHA, T. J. F.. Sistema Brasileiro de Classificação de Solos. 5 ed. Brasília: Embrapa, 2018.

SANTOS, L. A. C.; CAMPOS, M. C. C.; AQUINO, R. E.; BERGAMIN, A. C.; SILVA, D. M. P.; JUNIOR, J. M.; FRANÇA, A. B. C.. Caracterização de Terras Pretas Arqueológicas no Sul do Estado do Amazonas. Revista Brasileira de Ciência do Solo, Viçosa, v. 37 n.4, 2013. DOI: https://doi.org/10.1590/S0100-06832013000400001

SCHELLEKENS, J.; ALMEIDA-SANTOS, T.; MACEDO, R. S.; BUURMAN, P.; KUYPER, T. W.; VIDAL-TORRADO, P.. Molecular composition of several soil organic matter fractions from anthropogenic black soils (Terra Preta de Índio) in Amazonia: A pyrolysis-GC/MS study. Geoderma, v.288, p.154-165, 2017. DOI: https://doi.org/10.1016/j.geoderma.2016.11.001

SILVA, A. C. S.. Avaliação da fertilidade de solos antrópicos (Terra Preta de Índio) com ênfase na biodisponibilidade do fósforo. Dissertação (Mestrado em Agricultura e Sustentabilidade na Amazônia) - Universidade Federal do Amazonas, Manaus, 2006.

SILVA, F. W. R.; LIMA, H. N.; TEIXEIRA, W. G.; MOTTA, M. B.; SANTANA, R. M.. Caracterização química e mineralogia de solos antrópicos (Terras Pretas de Índio) na Amazônia central. Revista Brasileira de Ciência do Solo, Viçosa, v.35, n.3, 2011. DOI: https://doi.org/10.1590/S010006832011000300002

SOARES, M. D. R.; CAMPOS, M. C. C.; CUNHA, J. M.; MANTOVAVANELLI, B. C.; OLIVEIRA, I. A.; FILHO, E. G. B.; LEITE, A. F. L.. Variabilidade espacial da estabilidade dos agregados e matéria orgânica do solo em terra preta arqueológica sob pastagem. Gaia Scientia. v.12, p.125-133, 2018.

SOARES, R.. Agregação e distribuição da matéria orgânica em solos de terra preta de índio da Amazônia Central. Dissertação (Mestrado em Geoquímica Ambiental) Universidade Federal Fluminense, Niterói, 2007.

SOMBROEK, W. G.; RUIVO, M. D. L.; FEARNSIDE, P. M.; GLASER, B.; LEHMANN, J.. Amazonian Dark Earths as carbon stores and sinks. In: LEHMAN, J.; KERN, D. C.; GLASER, B.; WOODS, W.. Amazonian Dark Earths: Origin, Properties, and Management. Dordrecht: Kluwer Academic Publisher. 2003, p.125-139.

SOUSA, M. A.; REIS, I. M. S.; ALMADA, A. P.; ROSSI, C. Q.; PEREIRA, M. G.; PINTO, L. A. R. S.; SILVA, C. F.; SANTOS, O. A. Q.. Atributos químicos e frações da matéria orgânica em solos antrópicos na Amazônia Oriental. Brazilian Journal of Development, Curitiba, v.6, n.5, p.29623-29643, 2020. DOI: http://doi.org/10.34117/bjdv6n5-424 
TEIXEIRA, P. C.; DONAGEMMA G. K.; WENCESLAU, A. F.;

TEIXEIRA, G.. Manual de métodos de análise de Solo. 3 ed.

Rio de Janeiro: Embrapa Solos, 2017.

VIANA, S. F.; CAMPOS, M. C. C.; CUNHA, J. M.; NASCIMENTO

W. B.; JÚNIOR, A. F. M.. Variabilidade espacial dos atributos químicos em terra preta de índio cultivada na região de Novo Aripuanã, AM. Revista Verde de Agroecologia e

Desenvolvimento Sustentável, Pombal, v.11, n.3, p.53-58, 2016. DOI: https://doi.org/10.18378/rvads.v11i3.4385

A CBPC - Companhia Brasileira de Produção Científica (CNPJ: 11.221.422/0001-03) detém os direitos materiais desta publicação. Os direitos referem-se à publicação do trabalho em qualquer parte do mundo, incluindo os direitos às renovações, expansões e disseminações da contribuição, bem como outros direitos subsidiários. Todos os trabalhos publicados eletronicamente poderão posteriormente ser publicados em coletâneas impressas sob coordenação da Sustenere Publishing, da Companhia Brasileira de Produção Científica e seus parceiros autorizados. Os (as) autores (as) preservam os direitos autorais, mas não têm permissão para a publicação da contribuição em outro meio, impresso ou digital, em português ou em tradução. 